\title{
POTENTIA DEI: DE TOMÁS DE AQUINO A HOBBES
}

\begin{abstract}
Dra. María L. Lukac de Stier*
La intención del presente trabajo es analizar las consecuencias filosófico-teológicopolíticas que la distinción entre potentia dei absoluta y potentia dei ordinata, surgida en el medioevo, generará en el pensamiento político moderno, particularmente en el de Thomas Hobbes. Se parte de la consideración del tema que hace Pedro Damián. Luego se analiza en Tomás de Aquino la noción de potencia divina, el tratamiento que el Aquinate da a la omnipotencia y, finalmente, el valor secundario que Tomás asigna a la distinctio. Se continúa con el análisis de las versiones ofrecidas por Duns Scoto y Ockham. Se examina también la elaboración que de la omnipotencia divina hicieran Bonifacio VIII y Egidio Romano tal que permitiera explicar la relación del poder papal (plenitudo potestatis) con las leyes eclesiásticas y con el poder temporal. Se concluye con el tratamiento de la cuestión en la teología política de Thomas Hobbes, quien anula la distinctio en favor del poder absoluto.
\end{abstract}

Palabras clave: potentia absoluta, potentia ordinata, plenitudo potestatis, Tomás de Aquino, Thomas Hobbes.

\section{POTENTIA DEI: FROM THOMAS AQUINAS TO HOBBES}

The intention of the present work is to analyze the philosophical-theological-political consequences that the distinction between potentia dei absoluta and potentia de $i$ ordinata, emerged in the Middle Ages, will create for the modern political thinking, particularly in that of Thomas Hobbes. It's started from the consideration made by Pedro Damian. Then, the notion of divine power is analyzed in Thomas Aquinas, as well as the treatment that he gives to omnipotence and, finally, the secondary value he gives to the distinctio. It continues with the analysis of the versions offered by Duns Scotus and Ockham. It is also examined the elaboration made by Boniface VIII and Giles of Rome on divine omnipotence so it allows to explain the relationship of the papal power (plenitudo potestatis) with the ecclesiastic laws and temporal power. It concludes with the treatment of the problem in Thomas Hobbes' political theology, in which the distinction is overruled in favor of the absolute power.

Keywords: potentia absoluta, potentia ordinata, plenitudo potestatis, Thomas Aquinas, Thomas Hobbes.

\footnotetext{
*Universidad Católica Argentina-CONICET, Buenos Aires, Argentina. Correo electrónico: mstier@fibertel.com.ar
} 



\section{Introducción}

Mucho SE HA ESCRito, DESDE DifERENTES ENCUAdRes teológicos, SOBRe la omnipotencia divina. Mi intención, al abordar este tema, es analizar las consecuencias filosófico-teológico-políticas que la distinción entre potentia dei absoluta y potentia dei ordinata, surgida en el medioevo, generará en el pensamiento político moderno, particularmente en el de Thomas Hobbes. Si partimos de la conocida aseveración de Carl Schmitt de que "todos los conceptos sobresalientes de la moderna teoría del Estado son conceptos teológicos secularizados" ${ }^{\prime \prime}$ comprenderemos la importancia de analizar el concepto de omnipotencia divina en su distinción entre potentia absoluta y potentia ordinata.

Si bien la dupla absolutus/ordinatus tiene, estrictamente, su origen en la distinción jurídica entre quienes morían sin determinar sus últimas voluntades (absolutus) y quienes habían hecho testamento (ordinatus) ${ }^{2}$, el primer texto teológico en el que aparece la distinción entre dos formas de definir la omnipotencia divina le pertenece al reformador de la vida monástica del siglo XI, Pedro Damián ${ }^{3}$, que reporta su conversación durante una cena con su amigo, el abad Desiderio, en el monasterio de Monte-Cassino, probablemente en el año 1067. El diálogo versó en torno a las palabras de San Jerónimo quien, al recomendar el estado de virginidad en su Epistula ad Eustachium, afirmaba que incluso Dios, que puede hacer todas las cosas, no puede restaurar la virginidad a quien la ha perdido, ejemplificando así la imposibilidad de Dios de cambiar el pasado ${ }^{4}$. Mientras Desiderio no tenía dificultades para aprobar la afirmación de San Jerónimo, pues defiende la omnipotencia como el poder de hacer lo que sea que quiera hacer, parafraseando a San Agustín para quien Dios es llamado omnipotente porque es capaz de hacer todo lo que su voluntad quiere, Damián confiesa que no puede aceptarlas. Si lo hacía podía ser interpretado como que Dios solo puede lo que Él quiere y esto disminuiría el poder de Dios. Para Pedro Damián Dios puede hacer más de lo que de hecho quiere hacer; el poder divino no puede estar limitado por su voluntad ni por su naturaleza divina. Damián defiende la omnipoten-

1 Schmitr, C., Teología política. Cuatro ensayos sobre soberanía, Struhart \& Cía, Buenos Aires, 1998, p. 54.

2 Florido, Francisco León, "Providencia y libertad en la filosofía medieval latina" en Lombardo-Duns Escoto, Cuestiones sobre la omnipotencia divina, Escolar y Mayo, Madrid, 2011. Florido indica, a su vez, consultar el trabajo de Rand, E., "Potentia Dei conditionata una questione di Ugo si Saint-Cher sull'onnipotenza divina (Sent. I, 42, q.1)”, Rivista di storia della filosofia 39, Milán, 1984, pp. 521-553.

3 Damián, P., Disputatio super quaestione qua quaeritur si Deus omnipotens est, quomodo potest agere ut quae facta sunt facta non fuerint, en CANTIN, A., Pierre Damien: Lettre sur la toute-puissance divine (SC 191), Paris 1972, p. 406; este escrito es mejor conocido bajo el nombre de su edición más antigua De divina omnipotentia (PL 145, 595-622).

4 Epistula 22, 5 (CSEL 54, 150), cfr. Wright, F. A., (Ed.), Select Letters of St. Jerome (LCL), London, 1963, p. 62. 
cia trascendente de Dios aún cuando nunca ejerciera de hecho tal poder. De este modo se expresa contra aquellos que pretenden aplicar a los misterios de la divinidad los principios que rigen la lógica de la naturaleza, pretendiendo hacer del hombre la medida de las cosas divinas. Siguiendo a Damián, los teólogos posteriores tendieron a poner de relieve que, en un sentido absoluto, el orden natural está sometido a la voluntad divina.

Planteado el tema, en términos generales, pasemos al análisis del mismo tal como se encuentra en Tomás de Aquino. Antes de centrarnos en la cuestión de la omnipotencia analizaremos la noción previa de potencia divina que maneja Tomás. En el temprano Comentario a las Sentencias Tomás ofrece el concepto de potencia divina como resultado de diversas trasposiciones conceptuales. Leemos:

...La palabra potencia se impuso primeramente para designar la potestad del hombre, en cuanto que llamamos poderosos a algunos hombres como enseña Avicena...y después se trasladó a las cosas naturales. Parece que es potente aquel hombre que, sin impedimentos, puede hacer lo que quiere respecto de otros...Ahora bien la potencia de un agente natural o voluntario es impedida en la medida en que puede padecer la acción de otro agente. Por lo tanto, a la noción de potencia en su primera acepción corresponde el no poder padecer...y desde aquí es posible concluir que en Dios se da la noción perfecta de potencia tanto porque es activo respecto de todo [omnia agis]-y eso le corresponde en cuanto que es acto primero y perfectísimo-...-como porque nada padece-y eso le corresponde en cuanto que es acto puro que no se mezcla con ninguna materia 5 .

En el texto trascripto vemos cómo Tomás parte de la experiencia humana, pero también recurre a la autoridad de Avicena, de Agustín y de Aristóteles. Destacamos especialmente la influencia de la metafísica aristotélica del acto que es lo que, posteriormente, aparece en el tratamiento que Tomás ofrece del tema en la Suma Teológica. En la cuestión De divina potentia afirma que "hay una doble potencia: la pasiva, que en modo alguno está en Dios, y la activa, que es preciso atribuirle en grado máximo". Como Dios es acto puro le corresponde ser principio en grado máximo pues la razón de principio es aplicable a la potencia activa ya que ésta es principio de obrar sobre otro ${ }^{6}$. Encontramos un lugar paralelo en el De Potentia:

Pero a Dios le corresponde ser acto puro y primero; luego le pertenece en grado máximo el operar y el difundir su semejanza en otros y, en consecuencia, le compete en grado máximo la potencia activa ${ }^{7}$.

5 Tomás De Aquino, S., In I Sent., dist. 42, q. I, a.1, c.

6 Tomás De Aquino, S., S.Th., I, q. 25, a.1, c.

7 Tomás De Aquino, S., De Pot., q. 1, a.1, c. 
Como acertadamente sostiene el P. Ferrara, el acto aristotélico aplicado a Dios, "pasa por el doble tamiz de la remoción (acto puro) y de la eminencia (acto primero y perfectísimo). Por la remoción queda descartada de Dios toda potencia pasiva (Contra Gentes I, c.16, y II, c.25) y por la vía de eminencia se atribuye a la potencia divina toda la 'actuosidad' del acto, es decir, no solo el acto primero de la forma por el que se auto-constituye y se autodefine sino también el acto segundo de la operación o del actuar por el que se refiere a su objeto"8. Esta doble condición del acto, primero de la forma y segundo de la operación, fundamenta el doble significado de la potencia divina: el absoluto del esse y el relativo del operari. En el sentido absoluto la potencia divina se identifica con la actualidad pura de la esencia divina y con su infinitud. En el sentido relativo de la operación o del actuar la potencia divina se convierte en principio y causa de todo lo creado. Todo esto sin que se pierda la identidad entre el ser y el operar divinos.

Si pasamos ahora a la omnipotencia divina, llama la atención, como sabiamente advierte el P. Ferrara en el mismo trabajo, que Tomás al comentar el primer artículo del Credo explique todos los términos menos el de "Todopoderoso". Tampoco es la omnipotencia sino la providencia salvífica el núcleo de los artículos de la fe. En la cuestión "De Obiecto fidei" de la Suma Teológica, al analizar si los artículos de la fe están debidamente enunciados, se refiere a la omnipotencia sólo tangencialmente en la respuesta a la segunda objeción, señalando que Dios no podría obrar cuanto quisiera en los seres inferiores si no los conociera y no tuviera providencia de ellos ${ }^{10}$. También al referirse al nombre de Dios señala Tomás que en su origen significa la providencia sobre todos los seres ${ }^{11}$. Tampoco la omnipotencia es analizada al referirse a la naturaleza de Dios (qq.3-13) en el Tratado de Dios Uno, donde tienen prioridad la simplicidad, la bondad, la infinidad e inmensidad, la omnipresencia, la inmortalidad, la eternidad y la unidad. Esto es importante remarcarlo para contrastar con el lugar privilegiado que a la omnipotencia le concederán autores cono Duns Scoto y Ockham, cuya influencia pasará luego a la teología política moderna. En definitiva, Tomás trata el tema de la omnipotencia en la Cuestión Disputada De Potentia y en la Suma Teológica. En esta última aparece tratado como uno de los artículos (Utrum Deus sit omnipotens) de la cuestión De divina potentia, de la que se ocupa luego de haber analizado la ciencia y la voluntad divinas. En el mismo corpus del artículo señala la dificultad de encontrar la razón de la omnipotencia, ya que se presentan dudas de lo que está comprendido en el enunciado "Dios todo lo puede" 12 . Nuevamente recurrimos al P. Ferrara quien afirma que "la razón de la dificultad reside en aquel omne, aquella totalidad, que es objeto de la omnipotencia, porque se trata de una totalidad relativa, no se trata de una infinitud fuera de la cual nada hay sino de una infinitud fuera de la cual siempre cabe algo

8 Ferrara, Ricardo, "Infinitud y límites de la potencia divina en Santo Tomás de Aquino", Teología, 27, 1990, p. 10.

9 Cfr., Tomás De Aquino, S., Expositio in Symbolum Apostolorum, art. 1, Marietti, Torino, № 869-886.

10 Tomás De Aquino, S., S.Th, II-II, q. 1, a. 8, ad 2.

11 Tomás De Aquino, S., S.Th. I, 1.13, a. 8, c: "Cuantos hablan de Él llaman Dios al ser que tiene providencia universal de todas las cosas".

12 Tomás De Aquino, S., S.Th. I, q. 25, a. 3, c. 
más"13. Para Tomás lo más correcto sería entender que Dios puede todo lo posible y aclara que lo posible puede entenderse de dos modos, tal como lo afirma Aristóteles en Metafísica $\mathrm{V}, 12$ (Bk 1019b34): lo posible relativo a alguna potencia y lo absolutamente posible. Del primer modo sería posible para el hombre lo sujeto a la potencia humana y esto no puede aplicarse a Dios, pues no puede decirse que es omnipotente porque puede todas las cosas que son posibles para la naturaleza creada, ya que, por un lado, su potencia se extiende a mucho más y, por otro, Dios no puede pecar, ni fallar, ni morir. Añade Tomás que, si se dijese que es omnipotente porque puede todo lo que es posible para su potencia sería un círculo vicioso, pues equivale a decir que es omnipotente porque puede todo lo que puede. Concluye, entonces, que Dios puede todo lo absolutamente posible. Este posible absoluto resulta de la relación no contradictoria de los términos que lo enuncian. Esto significa: aquello cuyo predicado no repugna al sujeto. Por el contrario, el imposible absoluto es aquello cuyo predicado repugna al sujeto como que un hombre sea asno. Tomás ahonda todavía más el tema del posible absoluto indicando que todo lo que puede tener razón de ser está contenido entre los posibles absolutos. Y añade que nada se opone más a la razón de ser que el no-ser. Por lo tanto, lo único que repugna a la razón de absolutamente posible es lo que en sí mismo y, simultáneamente, implique el ser y el no-ser. Esto último es lo que no está sujeto a la omnipotencia, no por insuficiencia del poder divino, sino porque no puede ser posible. Por consiguiente, todo lo que no implica contradicción está entre los posibles respecto de los cuáles se dice de Dios que es omnipotente. Lo contradictorio no está contenido en la omnipotencia divina, pero más que decir que Dios no puede hacerlo, corresponde decir que no puede ser hecho ${ }^{14}$.

Volviendo, ahora, a las palabras de San Jerónimo que ocasionaron el diálogo entre Pedro Damián y Desiderio, Tomás las cita en el sed contra de S.Th., I, q. 25, a. 4 titulada Si Dios puede hacer que las cosas pasadas no hayan sido ${ }^{15}$. En el corpus responde que considerar que lo pasado no haya sido implica contradicción y, por tanto, no está sujeto a la omnipotencia divina. Es decir, continúa con el mismo argumento usado para explicar el posible absoluto. Pero en el ad tertium del mismo artículo vuelve a las palabras de San Jerónimo afirmando que Dios puede devolver a una mujer violada la integridad del alma y la del cuerpo, pero no puede quitarle que haya sido violada, como tampoco puede quitar a un pecador que haya pecado y perdido la gracia ${ }^{16}$. Es decir, los ejemplos solo amplían el argumento sostenido en el corpus y ubican la imposibilidad de que lo pasado no haya existido como imposible absoluto.

13 Ferrara, R., op.cit, p. 15.

14 Tomás De Aquino, S., S.Th., I, q. 25, a. 3, c. in fine. Cfr. De potentia, q. 1, a. 7, c.: "Dicendum ergo est,quod, sicut supra dictum est, potentia Dei, quantum est de se, ad omnia illa obiecta se extendit quae contradictionem non implicat..Relinquitur ergo quod Dei potentia ad ea se extendat quae sunt possibilia secundum se. Haec autem sunt quae contradictionem non implicant. Constat ergo quod Deus ideo dicitur omnipotens quia potest omnia quae sunt possibilia secundum se".

15 Tomás De Aquino, S., "Sed contra est quod Hieronymus dicit: Cum Deus omnia possit, non potest de corrupta facere incorruptam"(De custodia virginit.ad Eustach.epist., 22§5: ML22,397). Ergo eadem ratione non potest facere de quocumque alio praeterito, quod non fuerit".

16 Tomás De Aquino, S., S.Th., I, q. 25, a.4, ad 3. 
Por último, analizaremos en el pensamiento del Aquinate la distinción entre potentia absoluta y potentia ordinata. En la cuestión De divina potentia de la Suma Teológica, aparece la distinción en el ad primum del a.5: Si Dios puede hacer lo que no hace. La objeción sostenía que Dios no puede hacer lo que no ha previsto ni preordenado que había de hacer. Y no ha previsto ni preordenado qué habría de hacer sino lo que hace. Por lo tanto no puede hacer más que las cosas que hace. En la respuesta a la objeción Tomás primero aclara que en Dios la potencia, la esencia, la voluntad, el intelecto, la sabiduría y la justicia son una misma cosa. Por lo tanto nada puede haber en la potencia divina que no pueda estar en su voluntad justa ni en su entendimiento sabio. Sin embargo, como su voluntad no está determinada con necesidad a una cosa u otra, ni tampoco están determinadas la sabiduría y la justicia de Dios a este orden existente de cosas, nada prohíbe que en la potencia divina haya cosas que Dios no quiera y que no estén contenidas en el orden que estableció en el mundo. Aquí, antes de apelar a la distinción entre absoluta y ordinata, Tomás recuerda que la potencia se concibe como la que ejecuta, la voluntad como la que manda y la sabiduría y el entendimiento como los que dirigen. Entonces, sostiene Tomás, que respecto de lo que se atribuye a la potencia considerada en sí misma, en Dios se dice que lo puede por potentia absoluta, y se extiende a todo aquello en lo que se pueda salvar la razón de ente y evitar la contradicción. En cambio, lo que se atribuye a la potencia divina en cuanto ejecuta el imperio de su voluntad justa, se dice que Dios lo puede por potentia ordinata. Según esta distinción se debe afirmar que Dios puede hacer por su potentia absoluta cosas distintas de las que previó y determinó hacer. Sin embargo, por potentia ordinata no es posible que haga cosa alguna que no haya previsto y predeterminado, porque el mismo hacer se sujeta a la presciencia y a la predeterminación, no así el mismo poder que le es natural ${ }^{17}$. En conclusión, si Dios hace algo es porque lo quiere, ahora que pueda hacerlo no es porque lo quiera sino porque tal es el poder divino por su misma naturaleza.

Esta distinción entre potentia absoluta y potentia ordinata, que en Tomás tiene un valor secundario, se difunde entre los teólogos de la Universidad de París y, más tarde, los dominicos y franciscanos de la Universidad de Oxford generalizarán su uso. Tal como lo expresa Rivera García: "En este contexto, el poder absoluto aludirá a la inmensitas incomprehensibilis potentia Dei, a su completa libertad y a la contingencia del orden natural. En tanto que la potentia ordinata hará referencia a la acción comprensible de Dios, a los acontecimientos necesariamente derivados de sus leyes y a la estabilidad del orden natural"18. De todos modos, ninguno de los teólogos que empleó la distinción pensó que Dios tuviera dos poderes o potencias sino que se trata de dos modos de concebir o hablar acerca de un único poder divino ${ }^{19}$.

17 TomÁs De Aquino, S., S.Th., I, q.25, a.5, ad 1: “Quia ipsum facere subiacet praescentiar et praeordinationi: non autem ipsum posse, quod est naturale".

18 Rivera García, A., “Teología política: consecuencias jurídico-políticas de la Potentia Dei”, Daimon, Revista de Filosofía, No 23, 2001, p. 172.

19 Cfr. Van Den Brink, Gijsbert, Almighty God, A study of the doctrine of divine omnipotence, Pharos, KamperThe Netherlands, 1993, p. 72. 
Durante el siglo XIV el tema mismo de la omnipotencia divina toma protagonismo en las discusiones teológicas y filosóficas, en parte motivadas por la condena universitaria de 1277 en París. La comisión de teólogos reunidos por el obispo Tempier consideraba heterodoxo negar a Dios algún poder (Quod Deus non potest) como lo hacían los maestros aristotelizantes y averroizantes. Siguiendo la condena del naturalismo aristotelizante, Scoto va a darle un fundamento metafísico sistemático al voluntarismo y destacar la omnipotencia como el primero y más importante de los atributos divinos. Lo que a fines del siglo XIII aparece como una mera crítica al naturalismo aristotélico, se convierte en una doctrina sistemática centrada en la noción de potentia Dei absoluta, que tendrá insospechadas consecuencias en la teología política moderna. Las dos principales versiones de la distinctio absoluta/ordinata la ofrecen, precisamente, Duns Scoto y Ockham. Algunos intérpretes denominan clásica la versión de Ockham, que sigue en esto a Tomás, y jurídica la versión de Scoto, compartida por nominalistas como Robert Holcot, Adam Wodeham o Pierre d'Ailly ${ }^{20}$. Ockham, con un lenguaje más filosófico que jurídico, establece un paralelismo, por una parte, entre el poder absoluto y la potentia de iure, por otra parte, entre el poder ordenado y la potentia de facto. Para Ockham, para quien tal distinción no es real sino solo intelectual, Dios puede realizar de potentia absoluta lo que no hace de facto o de potentia ordinata ${ }^{21}$. Duns Scoto, en cambio, haciendo uso de la terminología jurídica invierte la teoría de Ockham, distanciándose también del Aquinate, cuando identifica el poder absoluto de Dios con el poder de facto, y el poder ordenado con su potentia de iure $^{22}$. Dios actúa de iure siempre que respete el cauce ordinario de la ley revelada, y de potentia absoluta cuando infringe de facto las normas naturales, sea cuando obra milagros o bien cuando quiere implantar otra lex recta u ordinaria. Scoto establece la similitud entre el poder divino y el poder de los reyes, señalando que la potentia absoluta y la ordinata no son incompatibles, si bien la ordinata se subordina a la absoluta. En el decir de Rivera García, "Dios aparece con los rasgos de un monarca omnipotente y capaz de sustituir en cualquier momento la ley por otra igualmente justa. Todo cambio derivado de su potentia absoluta supone, en suma, la instauración de un nuevo orden justo" ${ }^{23}$. Lo interesante en el caso de ambos autores, tanto en Scoto como en Ockham, es la utilización de la distinctio refiriéndola a la política. En el caso de Ockham el poder del Papa (Juan XXII) y en el caso de Scoto al usar, en su exposición de la Ordinatio, la analogía entre la figura de Dios y la de un rey para dilucidar la posible salvación de Judas, pues, para Scoto es posible que Dios pueda cambiar los hechos del pasado modificando el orden instituido por Él mismo por su potentia absoluta ${ }^{24}$.

20 Cfr. OAkley, F., "The Absolute and Ordained Power of God in sixteenth and seventeenth century theology", Journal of the History of Ideas, 59, 3, 1998.

21 Оскнам, G. de, Quodlibet VI, q.1, art.1: "Posse aliquid quandoque accipitur secundum leges ordinatas et institutas a Deo, et illa dicitur Deus posse facere de potentia ordinata. Alter accipitur 'posse' pro posse facere omne illud quod non includit contradictionem fieri, sive Deus ordinaverit se hoc facturum sive non, quia multa potest Deus facere quae non vult facere".

22 Duns Scoto, Ordinatio I, d. 44, q. unica, r: "Et ideo non tantum in Deo, sed in omni agente libere-qui potest agere secundum dictatem legis rectae et praeter talem legem vel contra eam-est distinguere inter potentiam ordinatam et absolutam; ideo dicunt iuristae quod aliquis hoc potest facere de facto, hoc est de potentia sua absoluta, vel de iure, hoc est de potentia ordinata secundum iura". 
El Prof. Ivanovic en su esclarecedor trabajo De Potentia Dei: some Western and Byzantine Perspectives ${ }^{25}$ distingue dos modos de abordar el problema de la omnipotencia divina: la perspectiva bizantina y la escolástica occidental. En el caso de Bizancio, el análisis del tema se centró en la naturaleza divina y sus atributos, sin dar lugar nunca a una connotación política. Ivanovic lo atribuye a la clara distinción entre el poder político del emperador y el poder espiritual de cada patriarca, que está limitado a su iglesia sin poder pretender influencia alguna en el territorio de otra $^{26}$. Para los bizantinos el tema de la omnipotencia divina quedaba perfectamente definido en las Sagradas Escrituras y, al no tener razones políticas, no sintieron necesidad alguna de elaborar distinciones posteriores como la que estamos tratando en este trabajo. Por el contrario, el cristianismo romano, centrado en la figura del Pontífice que reunía tanto el poder espiritual como el político, llevó a la escolástica occidental a desarrollar una elaboración del tema de la omnipotencia divina que permitiera explicar la relación del poder papal (plenitudo potestatis) con las leyes eclesiásticas ${ }^{27}$. La relación del poder temporal con el poder espiritual y la primacía de éste sobre aquél fue una temática muy frecuente, primero en la tradición canonística y, luego, en diferentes documentos pontificios. Pero hay uno en particular, la bula Unam Sanctam de Bonifacio VIII, que es "no sólo uno de los textos más paradigmáticos de la eclesiología política medieval, sino también una de las expresiones más logradas de la doctrina de la primacía papal" 28 . De hecho, ha sido caracterizada como la bula más famosa de la Edad Media. Bonifacio VIII durante su pontificado, entre 1295 y 1303, debió enfrentarse con cambios políticos en los reinos y principados europeos que empezaban a poner en duda la primacía del poder espiritual. Esto motivó que el papa promulgara una serie de bulas destinadas a definir la relación del papado con el poder temporal, como reacción, fundamentalmente, frente a la política del rey francés Felipe IV, el Hermoso, quien representaba el espíritu de las nacientes monarquía nacionales. En 1296 se promulga la bula Clericis laicos que niega toda superioridad del poder temporal sobre el clero, denunciando que el pago de tributos por parte del clero a la monarquía supone una relación de dependencia del clero respecto del poder temporal. Con la bula Antiquorum habet fida, promulgado en febrero de 1300, en coincidencia con el Jubileo declarado por Bonifacio, se sostiene la plenitudo potestatis papal, si bien en términos vagos, sin fundamentación teórica. En la Apostolica sedes, promulgada en mayo de 1300 se sostiene el origen papal de la autoridad temporal. En una exhortación dirigida directamente contra Felipe IV, la Ausculta fili, promulgada en 1301, Bonifacio sostiene que el papa ha sido instituido por Dios como juez supremo de todos los hombres; que ha sido colocado sobre reyes y reinos para actuar en Su nombre y, por tanto, el poder real está sujeto al papa. Finalmente, en noviembre de

23 Rivera García, A., op. cit., p. 172.

$24 C f r$. Duns Scoto, Ordinatio I, d. 44, q.unica, 11.

25 Ivanovic, Filip, "De Potentia Dei: Some Western and Byzantine Perspectives”, The European Legacy, Vol.13, $\mathrm{N}^{\mathrm{o}} 1,2008$, pp. 1-11.

26 Ibid., p. 4.

27 Ibid., p. 2.

28 Bertelloni, F., "Sobre las fuentes de la Bula Unam Sanctam", Pensiero Politico Medievale II, Patron Editore, Bologna, 2004, p. 101. 
1302 Bonifacio promulga la bula Unam Sanctam, que si bien asume los argumentos de las bulas anteriores, desarrolla con profundidad teológica y metafísica el tema de la subordinación de la espada temporal a la espiritual (gladius sub gladio ${ }^{29}$, no sólo como dato revelado sino también como afirmación filosófica que surge de la constitución ontológica de la realidad, tal cual fue instituida por Dios. La bula sostiene que existen dos espadas, la temporal y la espiritual, y que ambas están en poder de Pedro como cabeza de la Iglesia. Vale decir que la Iglesia es el lugar originario donde todo el poder se concentra. En cuanto al ejercicio de ese poder, Bonifacio afirma que el gobernante temporal ejerce la espada temporal en favor de la Iglesia. Ésta ejerce de modo inmediato la espada espiritual. Pero el orden de subordinación entre ambas espadas o poderes se funda tanto en la revelación como en la estructura ontológica del mundo. De este modo Bonifacio sostiene que las cosas espirituales son ontológicamente anteriores a las temporales; que esta anterioridad implica una relación de dependencia causal de las cosas temporales respecto de las espirituales y, por último, que las cosas temporales son reconducidas, mediante intermediarios ${ }^{30}$, a las espirituales, que son su fundamento ontológico-metafísico ${ }^{31}$. Lo que llama la atención en esta bula es que Bonifacio no utiliza la expresión plenitudo potestatis, expresión acuñada por Egidio Romano cuya obra era bien conocida por Bonifacio, quien se refiere, sin embargo, a la primacía papal con la fórmula potestas Petri. Sigo aquí la explicación que sobre el tema ofrece Bertelloni en su bien documentado trabajo "Sobre las fuentes de la Bula Unam Sanctam", quien considera esta fórmula como una expresión más moderada, que busca el equilibrio y trata de mantener la dependencia del poder temporal respecto del espiritual, sin que esa dependencia implique la anulación ontológica del poder temporal. Bonifacio sabía que la expresión plenitudo potestatis significaría la reducción total del poder temporal al espiritual, implicando la anulación del carácter de poder legítimo de aquél y su consecuente usurpación, y éste no era su objetivo.

Obviamente, por lo antedicho, el tratado De ecclesiastica potestate (1301) de Egidio Romano, constituye la formulación más radical de la primacía papal, a la que identifica con una doctrina absolutista de la plenitudo potestatis elaborada sobre una base filosófica neoplatónica. El objetivo de Egidio es mostrar con argumentos filosóficos que la Iglesia está por encima de naciones y reinos, mostrar las relaciones entre el poder espiritual y el poder temporal y, finalmente, presentar la naturaleza del poder espiritual como poder absoluto $^{32}$. En el cuerpo del tratado Egidio se vale de un texto de Dionisio Pseudo-Areopagita que muestra cómo en toda la realidad tiene lugar una reductio de lo inferior a lo superior

29 “Oportet autem gladium esse sub gladio et temporalem auctoritatem spirituali subjici potestati”... "Nam cum dicat Apostolus:Non est potestas nisi a Deo; quae autem sunt, a Deo ordinata sunt [Rom.,13,1], non autem ordinata essent, nisi gladius esset sub gladio".

30 Mirbt, C., Quellen zur Geschichte des Papstums und des Römischen Katholizismus, Tübingen, 1924, p. 211: "et tamquam inferior reduceretur per alium in suprema. Nam secundum B.Dionysium lex divinitatis est, infima per media in suprema reduci. Non ergo secundum ordinem universi omnia aeque ac immediate, sed infima per media et inferiora per superiora ad ordinem reducuntur".

31 Para profundizar el tema ver el riguroso y analítico trabajo de Francisco Bertelloni, "Sobre las fuentes de la Bula Unam Sanctam", ya citado.

32 Egidio Romano, De Ecclesiastica Potestate, I, cap. IV, ed. Richard Schols, Weimar, 1929. 
mediante intermediarios. Egidio aplica esta reductio a las relaciones de subordinación de la espada temporal a la espada espiritual, concluyendo que la temporal está ordenada a la espiritual según la estructura metafísica de la mediación ${ }^{33}$. Establece aquí una analogía entre el modo como Dios gobierna el mundo y el modo como la espada espiritual gobierna a los hombres, es decir, del mismo modo como Dios puede producir, sin causas segundas, los mismos efectos que puede producir con ellas, la espada espiritual puede producir, sin mediación de la espada temporal, los mismos efectos que puede producir con su mediación. Sin embargo, la existencia de la espada temporal no es superflua y se justifica porque en situación de normalidad, la espada temporal puede hacer en la realidad, de modo más conveniente, lo que la espada espiritual puede hacer directamente en la realidad. Egidio introduce aquí la diferencia entre "esencia" del poder y "modo" de ejercicio del poder. La "esencia" del poder está vinculada a la espada espiritual que puede todo y se extiende ad omnia. En cambio, la legitimidad de la espada temporal, en su orden temporal, concierne solamente al "modo" de ejercicio del poder. En casos de normalidad, para Egidio, es conveniente que las dos espadas cogobiernen. Pero esta situación se altera cuando irrumpe el casus imminens. Éste es un caso excepcional en el que caduca la legitimidad de la espada temporal y todos los poderes son reducidos a su origen, es decir, a la espada espiritual. En el casus imminens es donde la plenitudo potestatis papal alcanza su máxima expresión. "El paradigma de la plenitudo potestatis es el poder divino en el que está concentrado el poder de todo agente: in ipso Deo est plenitudo potestatis" ${ }^{34}$. Según Bertelloni, Egidio establece así una analogía entre teología y política, entre el obrar de Dios en el mundo mediante el milagro y el obrar de la plenitudo potestatis papal de la Iglesia. Egidio utiliza de este modo el binomio potentia Dei absoluta-potentia Dei ordinata, aplicándolo al Pontífice usando la expresión potestas absoluta y potestas regulata del papa ${ }^{35}$, que le permite comparar el obrar milagroso de Dios como expresión de la potentia Dei absoluta con el poder absoluto del papa cuando las cosas temporales son mal gobernadas, porque la espada temporal comete errores que afectan a lo espiritual implícito en las cosas temporales.

Desde luego la distinción entre potentia absoluta y potentia ordinata extendió su uso inicial atribuido al poder del Pontífice, al absolutismo de los reyes, permitiendo a papas y reyes apelar a la potentia absoluta cada vez que actuaran o quisieran actuar contra o fuera de la ley. Un ejemplo de esto es la apelación de Enrique VIII pidiendo al Papa que anule su primer matrimonio usando el derecho que éste tenía por su potentia absoluta ${ }^{36}$. Estos desarrollos escolásticos del tema de la omnipotencia divina facilitaron la aparición de la teología política a fines del medioevo, que se continuó en la temprana modernidad, ya influida por la Reforma.

33 Idem.

34 Cfr. Bertelloni, F., op. cit., pp. 106-113.

35 Cfr. OAKley, Francis, "Jacobean Political Theology: The Absolute and Ordinary Powers of the King", Journal of the History of Ideas, Vol. 29, ํㅜ 3, University of Pennsylvania Press, 1968, pp. 323-346.

36 Ivanovic, Filip, "De Potentia Dei: Some Western and Byzantine Perspectives", The European Legacy. Vol. 13, $\mathrm{N}^{\mathrm{o}} 1,2008$, p. 9. 
Así llegamos a una figura clave en la temprana modernidad, Thomas Hobbes, quien construye al decir de Carlo Altini, "un modelo teológico-político sobre una base atea y materialista" ${ }^{37}$. Si alguien duda que pueda hablarse de una teología en Hobbes recuerdo que, prácticamente, la mitad del Leviathan está dedicado a desarrollar el tema del papel de Dios en un Estado Cristiano ${ }^{38}$. Hobbes reconoce el derecho natural de Dios de reinar sobre los hombres, el cual se deriva no de su condición de Creador, sino de su "irresistible poder" 39 . Para el filósofo inglés "la naturaleza de Dios es incomprensible; no entendemos nada de lo que Él es, sino sólo que Él es, por tanto los atributos que le asignamos no dicen qué es Él, ni significan nuestro conocimiento de su naturaleza, sino sólo nuestro deseo de honrarlo con aquellos nombres que concebimos como más honorables entre nosotros" ${ }^{\prime 0}$. De estos atributos, para Hobbes, los más evidentes a nuestra razón natural son, en primer lugar, su existencia y, en segundo lugar, su irresistible poder. Tanto en el De Cive como en el Leviathan aparecen textos relativos a su existencia ${ }^{41}$ que han motivado a algunos intérpretes a señalar la presencia de un argumento ontológico. En cuanto al irresistible poder, es la clave hobbesiana para explicar el querer y la acción divinas que se identifican. Además, explica como la soberanía de Dios se deriva de su omnipotencia ${ }^{42}$. Pero, sobre todas las cosas, la omnipotencia para Hobbes es el modo de explicar la existencia de un ser incomprensible, pues hablar de un Dios que es, significa afirmar que existe un ser todopoderoso, cuyo poder es irresistible, que puede producir todas las cosas, en el cuál la omnipotencia se confunde con la incomprensibilidad. Así lo explica Martine Pécharman ${ }^{43}$ en una excelente exégesis del cap. I del "Apéndice" al Leviathan en latín, el que Hobbes enuncia la fórmula del símbolo niceno: "Credo in unum Deum Patrem omnipotentem factorem caeli et terrae, atque visibilium omnium et invisibilium" ${ }^{4}$.

Ahora bien, para Hobbes, ese poder irresistible de Dios es un poder absoluto. En su polémica con el obispo Bramhall, Hobbes sostiene "la suficiencia plena, cuando es atribuida

37 Altini, Carlo, La fábrica de la soberanía, El Cuenco de Plata, Buenos Aires, 2005, p. 112.

38 Personalmente creo que la exégesis bíblica y los argumentos teológicos expuestos en la tercera y cuarta parte del Leviathan responden: 1) a la forma habitual de tratar el tema de la autoridad en el s. XVII; 2) al modo que tenía Hobbes de debilitar las críticas que se alzaban en su contra, desde el sector cristiano romano e incluso del reformado anglicano y 3) a la posible intención de subvertir la doctrina política cristiana usando sus mismos términos. Reconozco que esta última interpretación es muy dura y como no se puede tener certezas de las intenciones, la dejo meramente enunciada.

39 Hoввеs, Тн., Lev. XXXI, E.W. III, p. 345: "The right of nature, whereby God reigneth over men, and punished those that break his laws, is to be derived not from his creating them, as if he required obedience as of gratitude for his benefits; but from his irresistible power".

40 Hobbes, Th., Lev. XXXIV, E.W.III, p. 383.

41 Hoвbes, Тн., De Cive, E.W.II, p. 213: "First, it is manifest that existence is to be allowed him, for there can be no will to honour him, who we think hath no being"; HoBвES, TH., Leviathan, E.W. III, p. 351: "first we ought to attribute to him existence. For no man can have the will to honour that, which he thinks not to have any being".

42 Hoввеs, Тн., Lev. XXXI, E.W. III, p. 346: "To those therefore whose power is irresistible, the dominion of all men adhereth naturally by their excellence of power; and consequently it is from that power, that the kingdom over men, and the right of afflicting men at his pleasure, belongeth naturally to God Almighty; not as creator and gracious; but as omnipotent".

43 Pécharman, Martine, "La puissance absolue de Dieu selon Hobbes", en Canziani-Granada-Zarka (ed.), Potentia Dei-L'onnipotenza nel pensiero dei secoli XVI e XVII, Franco Angeli, Milano, 2000, pp. 269-293.

44 Appendix ad Leviathan, C.I (De symbolo niceno), en O.L. III, p. 511. 
a Dios, no significa otra cosa que su omnipotencia; y la omnipotencia no significa más que el poder hacer todas las cosas que Él quiera. Pero, para la producción de cualquier cosa, la voluntad de Dios es tanto un requisito como lo son el resto de su poder y suficiencia. Consecuentemente, su suficiencia plena significa una insuficiencia o falta de poder para hacer aquellas cosas que no quiere"45. Analizando este texto Luc Foisneau marca tres aspectos a considerar: $1^{\circ}$ la razón suficiente es igual a omnipotencia; $2^{\circ}$ la omnipotencia es definida como el poder de hacer todo lo que uno quiere; $3^{\circ}$ no hay omnipotencia sin que esté involucrada la voluntad. El final del texto es inequívoco: no hay fundamento para garantizar a Dios el poder hacer cosas que Él no quiera. Para Foisneau esta afirmación anula la distinctio entre potentia absoluta y potentia ordinata. No sólo anula la distinctio, sino que transforma la relación entre poder y voluntad ${ }^{46}$. En lugar de someter el poder divino a la voluntad de Dios, como el Aquinate, Hobbes somete la voluntad al poder de Dios, lo que equivale a defender la doctrina de la necesidad absoluta. Para el filósofo inglés todo lo que existe tiene que ser un efecto necesario del poder divino, y Dios quiere aquello sobre lo que tiene poder.

Otro argumento que en la doctrina hobbesiana anula la distinctio y muestra la vinculación de este tema teológico con el político, es el rechazo que Hobbes establece a la posibilidad de que alguien se obligue a sí mismo, tal como se lee en el Leviathan al explicar lo que significa que el soberano sea legibus solutus. El soberano de un Estado no está sujeto a las leyes civiles porque teniendo el poder de hacerlas y derogarlas o abolirlas, cuando le place, es libre de toda sujeción a ellas. Pues es libre -afirma Hobbes-aquel que puede serlo cuando así lo quiere. Además - continúa- es absurdo pensar que una persona pueda obligarse a sí misma, porque el mismo que puede obligar puede relevarse de toda obligación, por lo que tal obligación no existe ${ }^{47}$. Este mismo argumento es el que Hobbes usa para oponerse al obispo Bramhall en su polémica, cuando éste le recuerda que "nada es imposible para el poder absoluto de Dios; pero de acuerdo a su poder ordenado, dispuesto por su voluntad divina, Él no puede cambiar sus propios decretos ni puede incumplir su promesa"48. Además, ese poder ordenado tiene como fundamento la obligación que Dios libremente se impone a sí mismo con respecto a las promesas hechas a los hombres. Hobbes rechaza estas afirmaciones apelando a un error de principio. Como bien advierte Foisneau, si se anula el concepto de obligación hacia uno mismo, el concepto de potentia ordinata Dei pierde su sentido originario ${ }^{49}$. Si tenemos en cuenta que Hobbes denomina al Estado ese "dios mortal" ${ }^{50}$, entenderemos que los mismos atributos y poder que atribuye al Dios inmortal se los atribuye al Leviatán. De este modo, su teología política se aparta,

45 HobBes, Th., The Questions concerning Liberty, Necessity and Chance, E.W.V, XXXI, 427. (Traducción propia)

46 Foisneau, Luc, "Omnipotence, Necessity and Sovereignity- Hobbes and the Absolute and Ordinary Powers of God and King", en Springborg, P., (ed.), The Cambridge Companion to Hobbes's Leviathan, Cambridge University Press, New York, 2007, p. 274.

47 HobBes, Th., Lev. XXVI, 2, E.W. III, p. 252.

49 Bramhall, J, Castigations of Mr. Hobbes his last Animadversions in the case concerning Liberty and Universal Necessity, en The Works of John Bramhall, Vol. 4,Oxford, John Henry Parker, p. 1844.

50 Folsneau, L., op. cit., p. 282. 
definitivamente, del anglicano derecho divino de los reyes, sostenido por James I, quien recurría a la distinctio para explicar su doble poder: el ordinario, por el cual el mismo soberano debe seguir sus propias leyes porque se ha obligado libremente a hacerlo; y el poder absoluto, por el que puede cambiar las leyes, afirmando su superioridad sobre las mismas. El esquema jacobino era perfectamente compatible con la doctrina de los canonistas medievales que justificaban la plenitudo potestatis del Papa, que por su potentia absoluta no estaba limitado por las leyes de la Iglesia, pero, a la vez, por su potentia ordinata se obligaba a sí mismo a respetarlas ${ }^{51}$.

Indudablemente, en la teología política hobbesiana tuvo una incidencia fundamental la teología de la Reforma, en sus representantes principales: Lutero y Calvino, quienes combatieron la distinctio como una inútil y peligrosa sutileza de los escolásticos, pero esto sería tema para otro artículo. La herencia de la Reforma hace mella en Hobbes que actúa como gozne entre el Medioevo y la Modernidad, pero, indiscutiblemente, su teología política se constituye en el fundamento de la teoría política moderna.*

\section{Bibliografía}

Altini, Carlo, La fábrica de la soberanía, El Cuenco de Plata, Buenos Aires, 2005.

Bertelloni, F., "Sobre las fuentes de la Bula Unam Sanctam”, Pensiero Politico Medievale II, Patron Editore, Bologna, 2004.

Bramhall, J., Castigations of Mr. Hobbes his last Animadversions in the case concerning Liberty and Universal Necessity, en The Works of John Bramhall, Vol. 4, John Henry Parker, Oxford, p. 1844.

DAmí́n, P., Disputatio super quaestione qua quaeritur si Deus omnipotens est, quomodo potest agere ut quae facta sunt facta non fuerint, en CANTIN, A., Pierre Damien: Lettre sur la toute-puissance divine (SC 191), Paris, 1972.

Egidio Romano, De Ecclesiastica Potestate, I, cap. IV, ed. Richard Schols, Weimar, 1929.

Ferrara, Ricardo, "Infinitud y límites de la potencia divina en Santo Tomás de Aquino", Teología, 27, 1990.

51 Hobbes, Th., Lev. XVII, E.W.III, p. 158.

52 Cfr. Rivera García, A., op. cit., p. 173. Para ampliar este tema puede verse Oakley, F., "Jacobean Political Theology: the Absolute and Ordinary Powers of the King", Journal of the History of Ideas, 29-1, 1968.

*Artículo recibido: 26 de octubre de 2012. Aceptado: 22 de diciembre de 2012. 
Florido, Francisco LeÓn, "Providencia y libertad en la filosofía medieval latina" en LomBARdo-Duns Escoto, Cuestiones sobre la omnipotencia divina, Escolar y Mayo, Madrid, 2011.

Foisneau, Luc, "Omnipotence, Necessity and Sovereignity- Hobbes and the Absolute and Ordinary Powers of God and King", en Springborg, P., (ed.), The Cambridge Companion to Hobbes's Leviathan, Cambridge University Press, New York, 2007.

HobBes, Thomas, Leviathan. , De Cive.

, The questions concerning liberty, necessity and chance.

Ivanovic, Filip, "De Potentia Dei: Some Western and Byzantine Perspectives”, The European Legacy, Vol.13, № 1, 2008.

Mirbt, C., Quellen zur Geschichte des Papstums und des Römischen Katholizismus, Tübingen, 1924.

OAKLEY, F., "Jacobean Political Theology: the Absolute and Ordinary Powers of the King", Journal of the History of Ideas, 29-1, 1968.

------------, "The Absolute and Ordained Power of God in sixteenth and seventeenth century theology", Journal of the History of Ideas, 59, 3, 1998.

Ockham, Guillermo De, Quodlibet VI.

Pécharman, Martine, "La puissance absolue de Dieu selon Hobbes", en CanZiani-GranAdA-Zarka (ed.), Potentia Dei-L'onnipotenza nel pensiero dei secoli XVI e XVII, Franco Angeli, Milano, 2000.

RAndi, E., "Potentia Dei conditionata una questione di Ugo si Saint-Cher sull'onnipotenza divina (Sent. I, 42, q.1)”, Rivista di storia della filosofia 39, Milán, 1984, pp. 521-553.

Rivera García, A., “Teología política: consecuencias jurídico-políticas de la Potentia Dei”, Daimon, Revista de Filosofía, № 23, 2001.

Schmitт, C., Teología política. Cuatro ensayos sobre soberanía, Struhart \& Cía., Buenos Aires, 1998.

Van Den Brink, Gijsbert, Almighty God, A study of the doctrine of divine omnipotence, Pharos, Kamper-The Netherlands, 1993.

Wright, F. A., (Ed.), Select Letters of St. Jerome (LCL), London, 1963. 